\title{
Politiques internationales de solidarité numérique en Afrique
}

African digital solidarity international policies

Políticas internacionales de soliridad digital en África

Destinity Tchéhouali

\section{CpenEdition}

\section{Journals}

Édition électronique

URL : http://journals.openedition.org/ctd/311

DOI : $10.4000 /$ ctd. 311

ISSN : 2491-1437

Éditeur

Chaire Unesco Pratiques émergentes en technologies et communication pour le développement

Référence électronique

Destinity Tchéhouali, « Politiques internationales de solidarité numérique en Afrique », Communication technologies et développement [En ligne], 5 | 2018, mis en ligne le 02 janvier 2018, consulté le 10 décembre 2020. URL : http://journals.openedition.org/ctd/311 ; DOI : https://doi.org/10.4000/ctd.311

Ce document a été généré automatiquement le 10 décembre 2020.

Communication, technologies et développement 


\title{
Politiques internationales de solidarité numérique en Afrique
}

\author{
African digital solidarity international policies \\ Políticas internacionales de soliridad digital en África
}

\author{
Destinity Tchéhouali
}

\section{NOTE DE L'AUTEUR}

\section{SWAHILI}

Muhtasari: Mahandiko hii inaonesha hisa za sera za kimataifa katika uwanja ya maendeleo ya digital katika nchi za Kusini kwa kuchunguza mipango ya ushirikiano ya digital uliofanywa kati ya 2009 na 2013 na Shirika la (ASN) na Fond Mondial de la solidarité numérique (DSF) pale Afrika. Mahandiko hii hutegemea kule mashirikisho uliofanywa katika mazingira ya utafiti « recherche-action » na vilevile katika mifano ya miradi ya digital na miradi ya telemedicine. Ile yote inaonyesha tufauti kubwa na tufauti kati ya majadiliano ya taasisi za kukuza ushirikiano wa kimataifa wa kimataifa, mu kikwanza, na malengo ya miradi au mipango yao, na matarajio ya umma wanaopata faida na matokeo halisi ya mafanikio ya umma. kutokana na utekelezaji wa miradi hii katika shamba.

Maneno: sera za kimataifa, maendeleo ya digital, Afrika, ushirikiano

1 L'expansion rapide des technologies numériques au cours de ces dernières décennies ne doit pas faire occulter que, dans la plupart des pays en voie de développement, notamment africains, il existe encore de nombreuses inégalités et fractures en matière d'accès et d'utilisation de ces technologies. Selon le nouveau rapport sur le développement dans le monde ${ }^{1}$, publié par la Banque mondiale, bien que le nombre d'utilisateurs d'Internet dans le monde ait plus que triplé depuis 2005, 4 milliards de personnes n'y ont pas accès, et $60 \%$ de la population mondiale reste encore exclue d'une économie numérique en constante expansion. Ce rapport insiste sur la nécessité de combler le fossé numérique persistant en misant sur des stratégies de développement 
numérique plus ambitieuses et en mettant en place des conditions favorables pour instaurer un cadre juridique et institutionnel qui permettrait à la technologie de procurer un maximum d'avantages au plus grand nombre. Par ailleurs, le dernier rapport "Mesurer la société de l'information ${ }^{2}$ » de l'Union internationale des télécommunications (UIT), publié fin 2015, révèle que l'Afrique est de loin la région où les niveaux de l'indice de développement des TIC $^{3}$ (IDI) sont les plus faibles (avec une valeur moyenne de 2,5) et où se situent la plupart des pays les moins connectés ( 29 sur les 37 que compte la région). L'UIT souligne que ces résultats illustrent à quel point l'Afrique est en retard sur le reste du monde en matière de développement des TIC et rappelle l'importance de remédier à la fracture numérique qui la sépare des autres régions.

2 Ces constats constituent un véritable aveu de l'échec des politiques internationales de solidarité numérique et de lutte contre la fracture numérique, qui n'ont pas été à la hauteur des attentes suscitées une dizaine d'années auparavant, lors des sommets mondiaux sur la société de l'information (SMSI), rendez-vous historiques d'une prise de conscience collective et d'une mobilisation sans précédent. Rappelons que c'est vers le milieu des années 1990 que la question de l'accès et de l'adoption des technologies de l'information et de la communication (TIC) dans les pays en voie de développement a commencé à susciter de nombreux débats sur la scène internationale. Au centre des préoccupations, la problématique des fractures numériques était principalement abordée sous l'angle des disparités en matière de connectivité, d'accès aux infrastructures et aux équipements, aspects considérés comme prioritaires dans la mise en œuvre des politiques et stratégies nationales de développement des TIC et de la société de l'information.

Dans ce contexte, les organisations internationales, et en particulier les agences et institutions spécialisées de l'ONU, ont été les premières à véritablement promouvoir la possibilité d'un rattrapage technologique, en préconisant notamment aux pays en voie de développement d'utiliser les TIC pour brûler les étapes du développement et d'accomplir ainsi un saut technologique (ou leapfrog) pour rattraper leur retard économique.

Dès 1998, la fracture numérique est devenue une priorité de l'agenda international à travers la décision de l'UIT de s'engager à trouver des solutions. Le sommet mondial sur la société de l'information (SMSI), en deux phases, en 2003 à Genève, puis en 2005 à Tunis, s'est caractérisé par l'implication de plusieurs parties prenantes, y compris des représentants de la société civile, dans les débats et le processus de prise de décision. En 2005, un Fonds (FSN, basé à Genève) et une Agence mondiale de solidarité numérique (ASN, basée à Lyon) ont été créés et ont reçu pour mandat de concrétiser la volonté commune des pays du Nord et du Sud à mettre en œuvre un pacte de solidarité numérique, en finançant et en mettant en œuvre des projets ou programmes internationaux en matière de lutte contre la fracture numérique Nord-Sud, en particulier dans des domaines tels que la connectivité, l'éducation numérique et la télémédecine. Or, après d'importantes difficultés financières et une crise de gouvernance institutionnelle, ces deux organisations ont dû être dissoutes quelques années seulement après leur création (le FSN en octobre 2009 et l'ASN en décembre 2011). Quelles sont donc les raisons qui expliquent cet échec des politiques et programmes internationaux de solidarité numérique? Le mode opératoire du FSN et de l'ASN par rapport aux transferts de technologie, d'expertise et de ressources était-il vraiment adapté aux différents contextes socioculturels, politiques et économiques des pays africains dans lesquels ces institutions intervenaient? En quoi les expérimentations et initiatives locales de solidarité numérique, notamment celles menées dans le cadre des partenariats de coopération 
décentralisée entre des collectivités françaises et des collectivités africaines, ont-elles pu produire des résultats plus significatifs que les programmes internationaux promus par l'ASN ou le FSN?

5 Notre méthodologie s'appuie sur l'exploitation des matériaux et des données d'enquête de terrain mobilisés dans le cadre de notre recherche doctorale, effectuée entre 2009 et 2013, sur l'évaluation des programmes et politiques internationaux de solidarité numérique. La méthode de construction des énoncés dans cette recherche a été empruntée à l'approche préconisée par Jean-Pierre Olivier de Sardan ${ }^{4}$ sur l'émicité (autrement dit l'attention portée au point de vue des acteurs) et la descriptivité (autrement dit le recours à l'observation). Les analyses que nous livrons sont le fruit d'une démarche d'observation participante et d'un exercice de réflexivité critique réalisés dans un contexte de recherche-action dans laquelle nous avons dû assumer la double posture de chercheurpraticien $^{5}$. Bien que cette posture puisse paraitre un peu équilibriste, elle nous a permis d'accéder à des dynamiques de solidarité numérique observables aussi bien de l'intérieur que dans leur mouvance sur le terrain, avec des interactions ${ }^{6}$ et des logiques complexes entre les acteurs, mais surtout avec des écarts significatifs et des contradictions mis en évidence par la confrontation entre les discours promotionnels, les objectifs des programmes internationaux de solidarité numérique en Afrique et leurs résultats par rapport aux attentes des acteurs.

6 Ce travail présente dans un premier temps les raisons du fiasco de l'action internationale de solidarité numérique en Afrique, en partant de la rhétorique onusienne du rattrapage technologique pour déboucher sur le dysfonctionnement des institutions promotrices de la solidarité numérique, notamment le FSN et l'ASN. Dans un second temps, nous proposons un bilan critique des principaux programmes menés ou soutenus par ces institutions en Afrique, et nous expliquons pourquoi ces programmes ont échoué là où les initiatives locales de développement numérique, menées dans le cadre de la coopération décentralisée, ont connu plus de réussite.

\section{La vulnérabilité institutionnelle de la solidarité numérique internationale}

7 Nous avons pu identifier trois principaux facteurs de vulnérabilité institutionnelle ayant pu contribuer à déclencher la crise de gouvernance stratégique qu'ont subie l'ASN et le FSN. Il s'agit de :

i. la mauvaise gestion financière ;

ii. l'insuffisance des ressources et compétences humaines ;

iii. la faible capacité d'adaptation à l'environnement global.

8 Du point de vue de la gestion, la situation d'impasse financière décrite dans le rapport de situation présentée par le président du FSN, Alain Madelin, lors de la réunion extraordinaire du Conseil de fondation du FSN, le 27 janvier 2009 à Bamako, illustrait bien les importants dysfonctionnements du Fonds. Un constat témoignait à lui seul de cette mauvaise gestion : sur une période de trois ans, le Fonds a passé plus de temps à dépenser de l'argent en voyages pour tenter de «vendre » le $1 \%$ de solidarité numérique qu'à récolter de l'argent grâce à ce principe. Ainsi, sur les 6 millions d'euros encaissés par le Fonds (somme majoritairement constituée des contributions financières de ses États membres), 5 millions ont été dépensés dans le fonctionnement de la structure et 
seulement 1 million a été consacré à une dizaine de projets 7 . Étant donné que les statuts du Fonds (article 19-1) limitent à $6 \%$ maximum la part du budget qui devait être consacrée au fonctionnement, il s'agit là d'un large dépassement du pourcentage fixé, et donc d'une violation des statuts de l'organisation par le secrétariat exécutif du Fonds. Alain Clerc, secrétaire exécutif du FSN, est alors publiquement désavoué par Abdoulaye Wade, président d'honneur du Fonds, et Alain Madelin, qui lui ont reproché d'avoir mal géré et détourné des fonds. Dans la foulée, la démission du secrétaire exécutif est demandée et, quelques mois plus tard, en octobre 2009 à Dakar, le Conseil de fondation a dû prononcer la dissolution du FSN, qui n'était plus à même d'accomplir ses missions, après les mesures suspensives suite au rapport de l'audit indépendant sur la gestion financière et managériale du Fonds en janvier 2009. Le projet de refondation des statuts et de transfert du siège du FSN en Afrique, qui avait été souhaité par les participants à la réunion de Bamako en 2009, n'a jamais abouti par manque de leadership d'un pays africain pour conduire ce processus à son terme.

9 Cette dissolution du FSN a mis au grand jour la vulnérabilité de l'ensemble du système institutionnel de gouvernance de la solidarité numérique, un système qui pour certains ne cadrait pas avec « les schémas traditionnels du développement extra-déterminés ${ }^{8}$ » et pour d'autres, constituait « un alibi » destiné à reporter une décennie plus tard le constat que le fossé numérique était quasi infranchissable pour une partie de l'humanité9. L'ASN, qui fonctionnait jusque-là dans l'ombre du FSN, aurait pu saisir cette occasion pour se repositionner comme le nouveau leader de cette cause sur la scène internationale, s'est retrouvée, elle aussi, empêtrée dans une importante crise politico-financière. Elle n'a pu s'engager dans un chantier de restauration de la confiance des partenaires internationaux qui ont été déçus par le FSN et n'a jamais réussi à se défaire de l'image négative laissée par le Fonds sur l'échiquier international. À un moment où la solidarité numérique avait besoin d'un opérateur stratégique pour financer et accompagner les porteurs de projets sur le terrain, l'ASN a perdu sa légitimité auprès de ses propres bailleurs et partenaires financiers.

Bien qu'elle ait reçu en juin 2010 une ultime subvention de 150000 euros, accordée par la délégation interministérielle pour l'éducation numérique en Afrique (DIÉNA) sur un nouveau mandat visant à assurer la promotion et la mise en œuvre d'un programme de jumelages éducatifs numériques en Afrique, Sankoré, l'Agence n'a jamais réussi à regagner la confiance de ses mandataires, ni à se relever de ses difficultés financières avec un budget de fonctionnement pérenne. Face à une trésorerie largement déficitaire (due entre autres à l'ardoise de près de 195000 euros de factures impayées qu'elle traînait depuis fin 2008, suite à l'organisation de la conférence internationale de Lyon pour la solidarité numérique) et à défaut de propositions concrètes de restructuration, de redressement et de relance des activités, le tribunal de grande instance (TGI) de la Ville de Lyon prononça la liquidation judiciaire de l'Agence le 6 décembre 2011.

11 Un autre indicateur de la vulnérabilité institutionnelle de la solidarité numérique internationale était le manque de ressources humaines compétentes au niveau de l'ASN et du FSN. En effet, compte tenu de la vocation «mondiale» du positionnement de leurs activités, l'ASN et le FSN étaient sous-dimensionnés : cinq salariés pour l'ASN et huit pour le FSN. Il faut également préciser qu'au sein des effectifs de chacune de ces structures, on pouvait identifier au moins deux à trois postes relevant de la gestion administrative (assistant de direction, assistant de projet, responsable des affaires administratives et financières). Il n'y avait donc pas assez de compétences qualifiées, ayant une expérience 
confirmée de terrain, au sein du FSN, et surtout à l'ASN, en matière de coordination ou de mise en œuvre de programmes de lutte contre la fracture numérique. Comment prétendre, avec des structures de si petite taille, s'attaquer aux défis complexes et multifacettes de la fracture numérique à l'échelle mondiale ? Comment réussir à assurer un plaidoyer crédible de la solidarité numérique et en faire une cause internationale sans avoir les moyens d'une telle ambition?

Ces questions résument à elles seules le fait que le FSN et l'ASN n'aient pas réussi à relever le défi de l'internationalisation de leurs activités afin de témoigner d'une véritable légitimité et d'une bonne capacité d'adaptation dans leur milieu. En réalité, ces structures n'étaient pas à la hauteur de la vocation "mondiale" à laquelle elles prétendaient. Pour mieux s'adapter à leur environnement et acquérir la légitimité nécessaire, elles auraient dû concentrer leurs efforts et leurs stratégies sur le passage de l'internationalisation à la transnationalisation de leurs activités ${ }^{10}$. Pour Philippe Ryfman ${ }^{11}$ , un tel passage se caractérise par cinq axes principaux du développement d'une ONG, notamment :

i. la compétence opérationnelle alliée à l'expertise ;

ii. l'aptitude à élargir régulièrement le mandat ;

iii. des potentialités financières largement accrues et diversifiées;

iv. la capacité à mobiliser des soutiens dans les opinions publiques, combinée à l'aptitude au lobbying sur les centres de pouvoir et facilitée par la sympathie d'une partie des élites; (v) la volonté de construire de véritables rapports de force, mais aussi des partenariats plus équilibrés, avec des entreprises transnationales, des organisation internationales, des États.

\section{Bilan critique des programmes internationaux de solidarité numérique}

Nous proposons, dans cette partie, un regard critique sur deux principaux programmes internationaux de solidarité numérique dans lesquels se sont directement investis le Fonds et l'Agence, à savoir le programme Sankoré d'éducation numérique pour tous et le programme « 1000 unités de télémédecine en Afrique ».

Le programme d'éducation numérique pour tous : nouvel « éléphant blanc » de la politique internationale de solidarité numérique

14 Sankoré ${ }^{12}$ est un programme international de solidarité numérique dans le domaine de l'éducation, ciblant les 25 pays d'Afrique ${ }^{13}$ qui utilisent le français comme langue d'enseignement. Cette contribution française à la lutte contre la fracture numérique s'inscrit dans le cadre d'une mission confiée par Nicolas Sarkozy à Alain Madelin en 2008, alors que ce dernier était encore président du FSN. Le 23 janvier 2010, la commande politique se concrétise par la création de la DIÉNA par décret du Premier Ministre ${ }^{14}$. La DIÉNA a pour mission de coordonner le pilotage opérationnel du programme Sankoré.

La philosophie du programme repose sur l'idée selon laquelle l'évolution des technologies permet de réaliser aujourd'hui des classes numériques à très faible coût : un ordinateur personnel relié à un tableau numérique interactif (TNI) ou à un mur écran devenu interactif, par l'intermédiaire d'un vidéoprojecteur. L'enseignant, mais aussi les élèves peuvent écrire sur le TNI au moyen de stylets optiques ou directement avec le doigt (selon les matériels). Ils peuvent ajouter, modifier et effacer les éléments projetés à volonté, 
comme on le ferait sur un tableau noir. Le professeur reste au centre du dispositif. Les fondamentaux de l'enseignement, le face-à-face entre enseignant et élèves, demeurent. Les pays les plus pauvres peuvent être ainsi équipés et des ressources numériques interactives de qualité facilement diffusées, adaptées ou créées par les enseignants euxmêmes. Le projet se développe alors autour des principaux objectifs suivants :

i. promotion et diffusion massive de tableaux numériques interactifs dans les écoles africaines ;

ii. formation des enseignants au travers d'une université numérique francophone de formation des maîtres ;

iii. fédération d'une communauté d'enseignants créateurs de contenus, pouvant partager et adapter des ressources éducatives libres à leurs pratiques d'enseignement. À l'occasion du sommet Afrique-France de Nice (31 mai - 1er juin 2010), le programme Sankoré est présenté aux chefs d'État africains et a bénéficié d'une couverture médiatique sans précédent. Pour marquer les esprits, le message politique lancé par ses promoteurs se résumait ainsi :

«[...] Parce qu'avec le numérique, le savoir éducatif peut être facilement, librement et gratuitement partagé, parce que nous croyons à la créativité de tous et en particulier à celle des enseignants, parce que nous savons que cette créativité, mutualisée au travers des réseaux d'enseignants, peut contribuer aujourd'hui à faire vivre le rêve d'une meilleure éducation pour tous, nous avons imaginé Sankoré pour faire de ce rêve une réalité ${ }^{15}$ ".

En d'autres termes, avec ce programme, l'éducation numérique n'est plus réservée aux pays développés, mais elle se partage. Il suffit que le bon vieux tableau noir soit remplacé par un écran interactif, grâce à un équipement léger à faible coût (autour de 1000 euros), pour qu'enseignants et élèves des écoles africaines accèdent à toutes les richesses du patrimoine éducatif numérique. Le rêve vendu tient à peu de choses: des classes africaines, où l'instituteur, sans être expert informatique, dispense son cours avec des supports de présentation PowerPoint ou des images en 3D incorporés à un tableau blanc ou projetés sur un mur numérique interactif. L'instituteur peut non seulement écrire et effacer (comme sur un tableau noir), mais aussi afficher et modifier tout type de document (textes, images, sons, vidéos), et les enregistrer à l'aide d'un stylet qui fait office de souris. Le rêve tient dans une mallette (valise-pack) contenant un kit de classe numérique, offert à tous les chefs d'État africains présents à Paris à l'occasion de la célébration du 14 Juillet et du Cinquantenaire des indépendances.

Ce programme de solidarité numérique, tout comme les grands projets d'hôpitaux, de barrages, d'industries agroalimentaire... auxquels nous ont habitué les relations entre la France et l'Afrique, présentait toutes les caractéristiques d'un nouvel «éléphant blanc » inspiré du fantasme de l'éducation numérique comme nouvelle « recette-miracle » au mal-développement de l'Afrique. En effet, ce programme mettait l'accent principalement sur des offres d'équipement massif, contribuant de la sorte à une marchandisation et à une libéralisation du savoir et de l'enseignement. Ceci bénéficie sans doute plus à l'écosystème industriel de production et d'utilisation de ressources éducatives libres, pensé depuis les bureaux parisiens de la DIÉNA, qu'aux enseignants et élèves africains. La logique techno-marchande sur laquelle se fonde ce programme ainsi que son approche de contenus "clé en main» nourrissent l'ambition d'une "colonisation numérique culturelle» dictée par la compétitivité technologique des industries françaises spécialisées dans la fabrication d'équipements ou le développement de logiciels ludoéducatifs. Pour le professeur Alexis Dieth, 
« on se trouve en face de ce qui apparaît comme un énième projet d'acculturation planifiée, imposé de l'extérieur par des États qui ont une finalité d'expansion économique et culturelle. Si le projet de connecter l'Afrique au réseau mondial affiche clairement son objectif commercial qui est d'offrir des opportunités d'affaires aux opérateurs économiques africains, l'objectif de l'école numérique semble quant à lui, s'inscrire dans un projet plus vaste de diffusion culturelle $d u$ modèle économique libéral dominant ${ }^{16} »$.

Même s'il mise sur des opérations de jumelages numériques éducatifs pour intégrer une dimension de dialogue réciproque des cultures et des savoirs locaux/endogènes, le programme Sankoré, tel qu'il a été conçu et mis en œuvre, n'a pas apporté les résultats escomptés en matière de transformation pédagogique de l'école africaine par un usage ambitieux des TIC dans les systèmes éducatifs des pays d'Afrique francophone. Voici quelques éléments sur lesquels se fonde cette analyse et qui découlent des enseignements de notre démarche d'observation participante dans la phase pilote du programme, entre 2010 et 2011 :

- dans la phase pilote du programme Sankoré, la fabrication des ressources numériques n'intégrait pas les priorités des programmes scolaires nationaux des pays africains ciblés. Le processus même de création de contenus était confié à des éditeurs numériques de contenus pédagogiques en France (Paraschool, Val'Esti...). Les ressources incluaient des illustrations déjà fabriquées à l'étranger (France ou autres pays), pour lesquelles la DIÉNA s'était assurée de la libre utilisation ou reproduction en Afrique (achats forfaitaires ou don des producteurs concernés);

- au lieu de constituer de véritables coopératives nationales, fédérant les acteurs du système éducatif, pour soutenir la production de ressources locales et la numérisation de ressources pédagogiques existantes au niveau national de chaque pays bénéficiaire, la DIÉNA avait identifié et sélectionné arbitrairement des ONG et des associations (pas forcément spécialisées dans le domaine de l'éducation ou des TIC) pour servir de relais en tant que formateurs de formateurs dans les pays ayant signé un protocole d'accord pour participer au programme. Ces acteurs peuvent être considérés comme des meneurs locaux du jeu acculturatif, car ils sont activement mis à contribution dans le lobbying autour de la diffusion des tableaux numériques et des vidéoprojecteurs interactifs du programme Sankoré, dont ils sont censés être les ambassadeurs auprès des autorités gouvernementales africaines et au sein des communautés éducatives ;

- les objectifs de formation et d'accompagnement sur le long terme des maîtres, tels que prévus initialement par le programme, se limitaient dans la phase de mise en œuvre à des stages de formation ponctuels (de deux à cinq jours) organisés dans les écoles de formation des instituteurs (ÉFI) ou dans les espaces de l'Initiative francophone de formation à distance des maîtres (IFADEM). Ces stages étaient rarement complétés par une véritable mise en situation en présence d'élèves. Des centaines de kits de classe numériques furent ainsi livrés au Burkina Faso, au Mali, sans qu'aucune véritable formation ne permette aux enseignants de s'approprier ces outils. Cette situation suggère que peu d'enseignants utiliseront vraiment le matériel et qu'un bon nombre de ces kits ont dû rester soigneusement emballés ou rangés dans les bureaux des directeurs d'établissement;

- les ressources clé en main, livrées avec les tableaux numériques, sont souvent jugées trop décontextualisées et ne facilitent pas la mise en référence culturelle des enseignements liés aux curricula des pays africains participant au programme. À défaut d'une masse critique de contenus locaux, le projet s'est heurté à une résistance de la part des enseignants africains ;

- enfin, l'écosystème numérique du programme Sankoré n'intégrait pas des technologies plus traditionnelles telles que les livres et la radio, alors que des expériences antérieures avaient 
montré l'utilité et la pertinence de ces supports dans les programmes d'éducation et d'alphabétisation en Afrique. chez certains un enthousiasme démesuré par rapport aux potentialités techniques des outils et des équipements. Ils pensaient, par exemple, que les tableaux numériques interactifs révolutionneraient les méthodes d'enseignement en Afrique, ou que les ordinateurs individuels permettraient d'améliorer les rendements scolaires. Ils établissaient naïvement une supériorité absolue des méthodes numériques sur les outils traditionnels d'apprentissage et d'enseignement, en considérant qu'à chaque introduction d'un nouveau dispositif technologique en milieu scolaire, les élèves seraient souvent plus attentifs, plus motivés, car l'effet «nouveauté » de ces dispositifs aiguiserai leur curiosité et renouvellerait leur soif de découverte, leur envie d'apprentissage. D'autres acteurs pensaient au contraire que les outils techniques comportent des limites; ils peuvent se révéler contre-productifs du point de vue même des principes pédagogiques. Bastien Guerry, cofondateur de l'association OLPC France, nous confiait à ce propos que si l'enseignant africain n'use pas de créativité pédagogique, l'ordinateur ou le tableau numérique n'apporterait aucune plus-value, puisque « la pédagogie numérique ne peut pas simplement consister à exposer un enfant à un ordinateur ou à un tableau numérique. Si l'ordinateur est un véhicule, ce n'est pas lui qui nous dit où aller. L'ordinateur est certes un "amplificateur d'idées ", mais l'usage pédagogique de l'ordinateur nécessite de s'interroger sur les idées qu'on veut véhiculer ou transmettre avec. Car une fois que les pays reçoivent les tableaux numériques et que les enseignants ont appris à se servir des logiciels, il reste surtout à définir le rôle que ces tableaux auront dans la classe, à imaginer les interactions entre les professeurs, les élèves et à définir des programmes scolaires adaptés à ces nouveaux outils ${ }^{17}$ ».

Par ailleurs, certains enseignants africains expliquent leur attitude de rejet ou de nonutilisation des tableaux numériques par rapport à l'éventualité d'une panne technique sur le matériel sans qu'ils ne soient capables de la résoudre, la substitution de l'écran à l'écrit, des problèmes de luminosité et de positionnement des vidéoprojecteurs et du TNI (effets d'ombres portées constatées lorsque le vidéoprojecteur n'est pas fixé au plafond, manque de visibilité de certaines polices de caractères, placement du tableau trop haut ou trop bas pour la vue des élèves, etc.). Par ailleurs, ces nouveaux dispositifs ne font pas l'unanimité au sein même du milieu éducatif, car la plupart des enseignants que nous avons interrogés estiment que par rapport aux outils traditionnels, les supports numériques constituent une surcharge de travail dans la préparation des cours et exigent surtout un niveau minimum de maîtrise et de compétences numériques.

21 À titre d'exemple, un inspecteur de l'éducation au Bénin, se prononçant sur les projets d'équipement numérique de certaines écoles de l'agglomération de Cotonou dans le cadre du programme des jumelages numériques Sankoré, s'est plaint en expliquant que « les ressources éducatives numériques livrées avec les tableaux numériques sont des ressources importées, or il n'est pas encore prévu de formations à court terme pour permettre aux enseignants béninois de devenir des créateurs de leurs propres ressources numériques, adaptées au programme scolaire du pays ${ }^{18} »$. Au-delà de cette préoccupation de contextualisation, c'est surtout le problème de renforcement des compétences numériques qui est pointé, sachant que, dans ce genre de programme, le rôle de l'enseignant et l'exploitation du potentiel cognitif des TIC doivent rester prépondérants ${ }^{19}$. Or, les programmes tels que Sankoré s'inscrivent dans une démarche descendante, dite

Communication, technologies et développement, 5 | 2018 
top-down, qui néglige la prise en compte des besoins des enseignants, leur formation, le développement de ressources numériques au niveau local et l'intégration du dispositif dans les systèmes éducatifs nationaux.

\section{Le programme « 1000 unités de télémédecine pour l'Afrique »}

La télémédecine regroupe l'ensemble des pratiques médicales s'appuyant sur les télécommunications et les technologies numériques pour améliorer l'accessibilité aux soins de santé. Les projets de télémédecine proposent généralement cinq types de services :

i. la téléconsultation (un médecin donne une consultation à distance à un patient) ;

ii. la télé-expertise (un médecin sollicite à distance l'avis d'un ou de plusieurs confrères) ;

iii. la télésurveillance médicale (suivi préventif ou post-thérapeutique avec interprétation à distance de l'état de santé d'un patient) ;

iv. la téléassistance médicale (assistance portée à distance par un médecin à un autre professionnel de santé au cours de la réalisation d'un acte);

v. la régulation médicale (réalisation à distance d'un premier diagnostic pour déterminer des réponses adaptées).

Le programme " 1000 unités de télémédecine pour l'Afrique», l'un des importants chantiers du FSN, a été lancé le 5 mars 2008 à Genève par le président du Fonds, Alain Madelin. Ce programme ambitionnait de fournir à chaque hôpital de district en Afrique des outils diagnostiques modernes (échographie, électrocardiographie) et une connexion à Internet permettant des échanges en direct ou en différé avec des experts distants. Il prévoyait également la formation continue des professionnels de santé par e-learning. La mise en œuvre d'un tel programme était principalement motivée par l'ambition de pallier l'isolement des médecins africains et le manque de moyens diagnostiques au niveau des hôpitaux de district, deux problèmes majeurs. Mais il s'agissait surtout d'un argument électoraliste pour l'ancien président sénégalais Abdoulaye Wade, qui annonçait lors de la cérémonie de lancement du FSN en mars 2005 à Genève :

«[...] La télémédecine, au-delà de l'efficacité, est aussi une économie de moyens financiers, car là où on faisait 1000 images par semaine, on en fait 10000 et à un coût bien moins élevé. D'ici la fin de l'année 2005, tous les hôpitaux et établissements hospitaliers de Dakar seront connectés en réseau d'imagerie numérique, permettant non seulement une observation plus rapide, moins coûteuse et plus sécurisée contre les effets du temps, mais aussi d'avoir des échanges entre spécialistes sur un cas déterminé, par exemple entre les médecins du Sénégal et un ou plusieurs collègues du réseau situés en France ou en Suiss ${ }^{20}$ ».

Ce programme du FSN présentait certes des avantages en termes de déploiement d'infrastructures légères et facilement gérables au niveau local (connexion satellite VSAT et un ordinateur par médecin). Cependant, il n'apportait pas suffisamment de garanties ni en matière de formation continue des personnels de santé africains, ni en matière de prise en charge sur le long terme des coûts relatifs à la connexion satellitaire et à la maintenance des équipements. Par ailleurs, toute la communication du FSN s'était concentrée sur l'objectif chiffré des 1000 unités. L'accent était clairement mis sur l'équipement de masse, et le projet a alors pris la tournure d'une gigantesque opération de marketing humanitaire ou de charity-business, avec des appels lancés directement aux entreprises et aux collectivités locales françaises et européennes afin qu'elles financent une ou plusieurs unités de télémédecine à des coûts variant de 25000 euros (dans le cas 
de l'installation d'une unité fixe) à 45000 euros (pour une unité mobile sur camion). Dès son lancement, le projet s'est heurté à des réticences de la part des entreprises et des collectivités du Nord, surtout pour des raisons de coûts estimés excessifs.

Finalement, ce programme s'est soldé par des résultats très limités. En effet, sur les 1000 unités de télémédecine prévues, à peine dix (quatre au Burundi et six au Burkina Faso) ont été effectivement mises en place (soit $1 \%$ des prévisions du projet). Au Burkina Faso, le déploiement de l'unité de télémédecine dans la commune de Ouahigouya se présentait, au vu de ses résultats au terme de 24 mois d'opérations, comme l'un des projets pilotes de télémédecine les plus aboutis parmi la dizaine de projets coordonnés par le FSN. Dans cette localité, la connexion VSAT et les équipements informatiques mis à disposition par le FSN au profit des intervenants dans la lutte contre le VIH/sida (personnel médical, bénévoles) ont permis de réaliser une vingtaine de séances de téléconsultation et de téléenseignement par visioconférence entre les hôpitaux de la région et l'hôpital universitaire de Genève. Grâce au partenariat entre le FSN et le RAFT (Réseau en Afrique francophone pour la télémédecine), 62 personnes ont pu bénéficier de formations d'initiation à l'informatique et à la navigation internet, de formations dans la gestion de bases de données et de dossiers médicaux ou de stocks de médicaments, de formations d'utilisation avancée pour réaliser des consultations à distance et échanger des données... Pour assurer progressivement leur autonomie, les bénéficiaires du projet ont créé des activités génératrices de revenus grâce à la mise en place d'un cybercafé communautaire équipé de 20 ordinateurs.

Quel que soit l'intérêt qu'ils présentaient pour l'amélioration de l'accès aux soins et la formation des personnels de santé en Afrique, ces projets de télémédecine étaient confrontés dans leur mise en œuvre à des difficultés d'ordres financier et technologique ayant empêché leur déploiement à plus grande échelle. En effet, les contraintes relatives à la qualité des infrastructures de connectivité et aux coûts de leur maintenance ou de leur réparation ont posé de sérieux problèmes à la pérennisation de ces expérimentations. Nous ne disposons malheureusement pas à ce jour d'un bilan ou d'un rapport d'évaluation sur lequel nous appuyer pour apprécier l'impact concret ainsi que les effets positifs de ce programme sur l'amélioration du système sanitaire dans les localités pilotes de ce programme.

\section{Les initiatives locales de développement numérique par le biais de la coopération décentralisée : quels motifs de satisfecit?}

27 À l'échelle locale, nous pouvons définir la solidarité numérique comme l'ensemble des politiques et des formes d'action collective visant à optimiser la diffusion et l'appropriation des TIC par et pour les citoyens. Les partenariats de coopération décentralisée en matière de solidarité numérique s'appuient sur des stratégies ambitieuses de développement numérique qui mobilisent l'implication de différents partenaires et acteurs du développement territorial. Cette forme de solidarité numérique se veut participative, car elle établit une relation de confiance horizontale et une collaboration directe entre plusieurs types d'acteurs (la société civile et les collectivités territoriales, les administrations publiques locales, les agences locales ou régionales de développement de la société de l'information, les entreprises du tissu économique local 
opérant dans le secteur de l'informatique et des télécommunications). Les principales modalités de mise en œuvre à l'échelon local des actions de solidarité numérique sont l'assistance technique, le transfert de compétences et d'expertise, le financement de projets, la formation et le renforcement des capacités, le lobbying, le plaidoyer et la sensibilisation. Chargée de définir et de mettre en œuvre la stratégie du ministère des Affaires étrangères en matière de soutien et de développement des coopérations décentralisées, la délégation à l'action extérieure des collectivités (DAECT) agit à travers son dispositif d'appels à projets comme le principal bailleur financier des actions de solidarité numérique portées par les collectivités territoriales françaises ayant développé un savoir-faire en matière de lutte contre la fracture numérique. De 2006 à 2012, sept appels à projets, dont cinq annuels $(2006,2008,2009,2011$ et 2012) et deux triennaux (2007-2009 et 2010-2012), ont été lancés par la DAECT sur la thématique B4 « Lutte contre la fracture numérique - Solidarité numérique ». Ces appels thématiques visent à soutenir des projets qui favorisent l'utilisation des TIC dans les domaines tels que la gouvernance locale, l'apprentissage et la formation des jeunes, le développement économique, l'agriculture... Sur un total de 75 dossiers déposés en sept ans, 51 ont été retenus pour un cofinancement de la DAECT/MAEE (soit plus des deux tiers du nombre total de dossiers de candidature déposés). Les projets retenus représentent un coût de financement global de 3638971 euros. Ils peuvent être thématiquement regroupés en six secteurs d'intervention :

i. l'e-gouvernance et l'e-administration;

ii. l'éducation numérique et l'équipement informatique des écoles ;

iii. l'accès public et communautaire aux TIC et à Internet ;

iv. la formation et le renforcement des capacités TIC;

v. l'e-culture ou l'utilisation des TIC dans la culture;

vi. l'e-agriculture ou l'utilisation des TIC dans l'agriculture. Les secteurs de prédilection de l'action internationale des collectivités en matière de solidarité numérique sont principalement la gouvernance et l'administration électronique (18 projets), ainsi que l'éducation numérique et l'équipement informatique des écoles (15 projets).

Dans le domaine de l'administration électronique, la plupart des projets visent l'informatisation de l'état civil, des cadastres ou des actes domaniaux, la mise en réseau et l'interconnexion des services municipaux. Pour ce type de projets, l'objectif est généralement de doter les municipalités en matériel informatique ou en logiciels afin de les accompagner dans leur processus de numérisation ou de dématérialisation des actes ou des registres civils. On peut citer, à titre d'exemple, l'informatisation de la municipalité de Figuig au Maroc (coopération décentralisée avec le conseil général de Seine-Saint-Denis, projet financé en 2006), l'informatisation et l'interconnexion des cinq communes du Plateau au Bénin (coopération décentralisée avec l'agglomération du Grand Chalon, projet financé en 2006), l'informatisation des services municipaux de Cienfuegos (en coopération décentralisée avec la Ville de Saint-Nazaire, projet financé en 2006 et en 2010), ou encore l'informatisation des services municipaux de la mairie de Ntsoudjini (coopération décentralisée entre le conseil général de Seine-Saint-Denis et l'Association des maires de Ngazidja dans les Comores, projet financé en 2012).

Les projets menés dans le domaine de l'équipement informatique des écoles ont également la cote auprès des autorités locales du Nord et du Sud, puisqu'ils ont des retombées directes pour les populations bénéficiaires et donnent souvent lieu à des cérémonies d'inauguration qui permettent de promouvoir, à travers les médias et auprès 
de l'électorat, l'efficacité de l'équipe municipale en matière de coopération internationale. La Ville de Besançon s'est ainsi illustrée en 2006, puis en 2010, avec ses partenaires sénégalais (Dakar et Guinguinéo), respectivement à travers les projets Sénéclic et Guinguinéo.clic visant à fournir des solutions informatiques pour équiper les écoles primaires, les collèges et les lycées de ces localités. Soulignons que, depuis 2010, en dehors des projets "classiques» d'introduction de l'informatique en milieu scolaire (exemple de la coopération entre Limonest et Boura au Burkina Faso), le nombre de projets de coopération décentralisée s'appliquant spécifiquement à l'éducation numérique s'est multiplié, notamment grâce à des jumelages numériques entre les écoles et les lycées de Rosny-sous-Bois et de Cotonou (Bénin), de Malakoff et de Ngogom (Sénégal), et de Mantes-la-Jolie et de Matam (Sénégal).

Outre les projets d'e-gouvernance et d'e-administration locale et ceux d'éducation numérique, on dénombre neuf projets portant sur la création de lieux d'accès public aux TIC. C'est le cas de l'espace public numérique de Dissin, au Burkina Faso (porté par le département de La Manche), des centres multimédias municipaux d'Abu Dis, en Palestine, et de Kaya, au Burkina Faso (projets respectivement portés par la Ville de Rezé et la Ville de Châtellerault), ou encore de la bibliothèque numérique de la Ville de Granada, au Nicaragua (porté par le conseil général d'Indre-et-Loire en 2012). En ce qui concerne la formation et le renforcement des capacités TIC, seuls cinq projets sont recensés. En 2011, par exemple, Fontenay-sous-Bois a monté avec la Ville de Koungheul, au Sénégal, un programme dénommé «Koungheul : ville informatique » qui mise sur la formation de techniciens koungheulois à la maintenance informatique de ces matériels, en partenariat avec l'association Ateliers sans frontières.

31 Tous ces projets témoignent du fait que la coopération décentralisée offre un cadre privilégié pour passer des discours globaux sur la société de l'information à la mise en œuvre d'actions concrètes et opérationnelles de solidarité numérique selon une approche territorialisée. Elle offre aussi tant pour les villes du Nord que pour celles du Sud une perspective d'apprentissage de fonctionnement réticulaire et de développement de liens, de partenariats ou de solidarité en réseaux ${ }^{21}$. Cependant, la principale question que l'on est amené à se poser est de savoir quels sont l'efficacité et l'impact des actions menées.

À travers une enquête réalisée auprès des porteurs de projets (collectivités territoriales françaises) et de leurs partenaires bénéficiaires (collectivités africaines), nous avons cherché à savoir si les projets de solidarité numériques expérimentés dans le cadre de la coopération décentralisée présentent plus de résultats positifs que les vastes programmes d'éducation numérique et de télémédecine menés par le FSN et l'ASN.

Sur les 51 projets de coopération décentralisée recensés dans le domaine de la solidarité numérique, notre enquête bilan couvre la période 2006-2011 et concerne uniquement les 39 projets financés par le ministère des Affaires étrangères et du développement international (MAEDI) sur cette période. L'échantillon étudié permet déjà de refléter la diversité des modalités de mise en œuvre des partenariats, des types de difficultés ou problèmes sur le terrain, des facteurs clés de réussite... Nous avons enregistré 27 réponses (soit $69 \%$ de taux de réponses) au questionnaire d'enquête diffusé en ligne du 1er juillet au 31 décembre 2011. Ces réponses sont complétées par une dizaine d'entrevues semidirectes par téléphone. Parmi les répondants au questionnaire en ligne ( $47 \%$ de femmes et $53 \%$ d'hommes), on compte neuf directeurs ou responsables des relations internationales et de la coopération décentralisée / coopération internationale, dix chargés de mission relations internationales et coopération décentralisée, trois chargés 
de projets de coopération et de relations internationales, un chef de projet directeur des systèmes d'information (DSI), un adjoint au maire et deux conseillers municipaux, un président de comité de jumelage et du conseil des villageois. Les réponses de ces personnes ressources qui ont suivi, coordonné, voire piloté au sein de leur collectivité des projets de coopération décentralisée dans le domaine du numérique, ont été croisées avec des témoignages recueillis auprès de citoyens "ordinaires " (bénéficiaires directs des actions de solidarité numérique) ou d'autres acteurs de terrain intervenus comme des relais ou des intermédiaires dans les projets évalués.

\section{Le poids politique de l'engagement des collectivités dans des actions de solidarité numérique}

L'engagement des collectivités territoriales dans des actions de solidarité numérique commence symboliquement à partir du moment où l'autorité locale officialise la mise à l'agenda des TIC dans la politique de coopération décentralisée. La plupart des collectivités françaises interrogées affirment que la lutte contre la fracture numérique Nord-Sud est désormais un axe important de leur action internationale. Les TIC constituent ainsi des leviers d'actions transversales s'appliquant à des axes de coopération prioritaire pour les collectivités (éducation, formation et renforcement des capacités, appui institutionnel à la gouvernance locale, culture et tourisme...). Au-delà de l'opportunité d'exporter à l'international un savoir-faire local en matière de développement numérique, s'engager dans une action de solidarité numérique constitue donc, pour une collectivité française, le témoignage d'une véritable volonté politique de participation à l'édification d'une société de l'information plus inclusive et dont les bénéfices profitent équitablement aux territoires du Nord et du Sud. Les projets de solidarité numérique qui témoignent d'un véritable engagement politique de la collectivité misent ainsi beaucoup sur la mobilisation des élus, y compris ceux n'étant pas en charge de la coopération. Ces projets consacrent des moyens humains conséquents pour assurer leur suivi et accompagnement, prévoyant une formation spécialisée pour renforcer les capacités des agents au sein de l'équipe ( $46 \%$ de cas), un recours au service de consultants externes ( $36 \%$ de cas) ou un recrutement en interne d'un chargé de mission ou d'un stagiaire ( $18 \%$ de cas).

Le budget alloué peut varier sensiblement en fonction des objectifs et des ambitions que se fixent la collectivité, porteuse du projet, et son partenaire. En effet, d'après le recoupement de plusieurs témoignages des directeurs des relations internationales et de la coopération décentralisée, les postes de dépenses qui mobilisent le plus de ressources financières dans le budget global d'un projet de solidarité numérique sont, par ordre décroissant, l'achat d'équipements et de matériels, les missions de terrain et les déplacements dans le cadre du suivi et de l'accompagnement du projet, et, enfin, les dépenses liées à la formation et au renforcement des capacités.

\section{La qualité du partenariat Nord-Sud : quelles relations entre les porteurs et les bénéficiaires de projets de solidarité numérique?}

L'un des premiers éléments qui détermine la qualité d'un partenariat de solidarité numérique Nord- Sud est le choix du partenaire et les conditions dans lesquelles ce choix s'est effectué. Il ressort de notre enquête que la grande majorité des partenariats sont 
noués sur la base d'anciennes relations de coopération ou de jumelages. Les deux tiers des collectivités qui s'engagent dans un projet de solidarité numérique ont déjà été liées auparavant par une convention de coopération décentralisée. Soulignons également que la forte présence d'une population immigrée sur un territoire constitue un facteur pouvant inciter la collectivité en question à nouer des relations institutionnelles avec la collectivité du pays d'origine de ces immigrés. N'y aurait-il pas aussi, comme explication possible, le fait qu'une population immigrée présente sur un territoire constitue une forme d'incitation pour la collectivité en question à nouer des relations institutionnelles avec la collectivité du pays d'origine des immigrés ? La stratégie globale d'un partenariat de solidarité numérique qui se veut durable passe par une démarche de concertation, l'élaboration conjointe d'un diagnostic des besoins numériques de la collectivité bénéficiaire et une évaluation de la capacité de la collectivité solidaire à pouvoir répondre à ces besoins en proposant une offre adaptée. Il importe ainsi que le porteur de projet ait une bonne connaissance socioculturelle du territoire du projet, afin de fournir des solutions technologiques (technologies, équipements, logiciels) ou de transférer des compétences adaptées aux besoins réels de la population. Par ailleurs, les gages d'un partenariat de long terme restent déterminés par l'implication en amont, pendant la mise en œuvre et en aval du projet, de personnes ressources de la diaspora ou de membres actifs d'associations de migrants résidant dans la collectivité du Nord, mais ayant conservé un lien avec leur territoire d'origine au Sud. Enfin, pour préserver l'équilibre du partenariat, les collectivités partenaires doivent, en amont, co-concevoir la programmation budgétaire de leurs actions afin que la question financière (en tant que question politique et condition majeure pour garantir l'autonomie du partenaire) ne constitue pas un nœud de distorsions pouvant dégrader les relations entre les deux partenaires.

\section{Quelle efficacité et quels impacts des actions menées?}

17 porteurs de projets sur les 27 répondants déclarent que les objectifs de leurs projets sont adaptés aux besoins des bénéficiaires; 11 d'entre eux estiment que les résultats obtenus sont insuffisants comparés aux objectifs initiaux. Quelques projets sont en décalage avec leur contexte socioculturel, politique et économique. C'est le cas notamment des projets de coopération décentralisée, guidés par des résultats à court terme (peu importent les moyens et la manière, seul le résultat compte) et s'inscrivant dans des agendas électoralistes, se fixant des objectifs purement quantitatifs.

Ces projets combinent des objectifs de solidarité numérique avec une approche d'action humanitaire conduite dans l'urgence et nécessitant plus de professionnalisme dans le montage et le suivi-évaluation du partenariat. Les projets, dans une démarche de solidarité numérique «conteneur» avec l'envoi d'équipements informatiques (ordinateurs pour informatiser les services municipaux au Sud ou tableaux numériques interactifs pour équiper les écoles et collèges africains), doivent particulièrement prévoir des dispositions de formation et d'accompagnement pour s'assurer que les équipements et services informatiques mis en place seront maintenus, utilisés et appropriés durablement, au-delà de la période de financement par les partenaires du Nord. Le développement d'usages et d'applications liés à l'e-administration (outils de démarche en ligne, informatisation de l'état civil, outils cartographiques SIG, logiciels libres appliqués à la gestion du cadastre ou du foncier...) constitue à ce jour la principale plus-value de 
" solidarité » dans les projets par les collectivités territoriales françaises. Si l'on ne doit pas sous-estimer l'impact des actions de formation et de capacitation des acteurs, il faut retenir que, du point de vue des bénéficiaires des projets, ce sont plutôt les actions de désenclavement numérique (accès aux réseaux et aux infrastructures TIC de base) et de don de matériels qui sont les plus appréciées.

Parmi les difficultés (facteurs d'échec) les plus fréquemment évoquées par les porteurs de projets, on retrouve, par ordre d'importance :

i. l'insuffisance des infrastructures de base (réseau électrique fiable, réseaux de télécommunication et de transports, etc.);

ii. le manque de formation des agents d'exécution et des référents techniques du projet au Sud ;

iii. (iii) l'absence de volonté politique ou le manque de sensibilisation des élus locaux à la problématique de la fracture numérique ;

iv. la maîtrise insuffisante du cycle de projet (non-respect du planning et du suivi technique du partenariat) ; (v) le manque de sensibilisation et de formation des populations bénéficiaires, qui ne comprennent pas l'intérêt et n'utilisent pas les équipements et services TIC proposés.

Quant aux facteurs clés de réussite, les différentes expériences ont démontré la nécessité de prendre en compte :

i. l'identification et l'implication en amont du projet des personnes ressources (acteurs locaux) susceptibles d'apporter une expertise locale ;

ii. la continuité du suivi technique du partenariat au sein de chacune des deux collectivités partenaires, favorisant la capacité d'appropriation puis d'adoption des méthodes et des outils par les partenaires selon les besoins futurs du projet ;

iii. le choix des solutions techniques les plus simples et efficaces dans le design du projet (plateforme technologique, logiciels libres, logiciels propriétaires, applications, supports...);

iv. l'étude de faisabilité (diagnostic territorial) et la répartition des responsabilités de chaque partenaire dès la phase initiale du projet; (v) l'investissement dans la formation pour développer au Nord et au Sud des compétences locales en matière d'ingénierie de projet numérique territorial.

41 In fine, le bilan sommaire des projets de coopération décentralisée mis en œuvre dans le domaine de la solidarité numérique entre 2006 et 2011 montre qu'il y a des motifs importants de satisfecit compte tenu des modestes moyens de financement qui ont permis de réaliser des avancées importantes en matière de plaidoyer, de sensibilisation, d'équipement et de formation aux TIC des collectivités locales dans 22 pays. Grâce à l'engagement des collectivités territoriales françaises et de leurs partenaires, des infrastructures locales de réseaux de communication ont pu être déployées, des administrations municipales ont pu être modernisées par les TIC (avec des gains de temps et l'optimisation des coûts de gestion, l'acquisition de logiciels libres et d'équipements informatiques...) et de nombreuses populations ont pu bénéficier de lieux d'accès public à Internet, de formations à l'utilisation des TIC et de l'accès à des contenus en ligne adaptés au contexte local. Les principaux enseignements découlant de notre bilan analytique peuvent se résumer ainsi :

- la qualité et l'efficacité des partenariats de coopération décentralisée en matière de solidarité numérique Nord-Sud peuvent être déterminées par la structuration et l'adaptation de l'offre du Nord par rapport aux besoins des partenaires du Sud. Il importe, en effet, que l'offre technologique ou méthodologique proposée par la collectivité du Nord 
s'affranchisse des logiques d'assistanat et prenne véritablement en compte les capacités de réception et d'autonomisation des partenaires du Sud ;

- pour passer d'une approche partenariale basée sur des processus d'assistanat à une approche basée sur l'autonomisation des bénéficiaires, les projets de solidarité numérique doivent s'appuyer sur des logiques d'action du bas vers le haut (bottom-up) afin de limiter les risques de dérive bureaucratique ou d'instrumentalisation entre collectivités porteuses de projet et collectivités partenaires ;

- l'éthique, la réciprocité, l'ouverture et la transparence constituent des éléments clés de la chaîne de valeurs des partenariats de coopération décentralisée en matière de solidarité numérique ;

- les collectivités françaises pourraient davantage impliquer les associations de migrants présentes sur leur territoire lors du montage, de la mise en œuvre ou du suivi-évaluation des projets menés en partenariat avec les territoires d'origine de ces migrants ;

- les partenariats public-privé, mettant à contribution des entreprises locales du secteur des TIC implantées au Sud, constituent une garantie de fournir de façon rapide et efficace des services de déploiement, de maintenance et de réparation d'équipements et de technologies adaptées aux besoins des populations locales.

\section{Conclusion} politiques et programmes internationaux de solidarité numérique Nord-Sud, notamment l'ASN et le FSN, ont échoué tant sur le plan de leur institutionnalisation et de la construction de leur légitimité que sur celui de la conception de leurs programmes et des résultats insignifiants comparés aux moyens et aux efforts consentis. Nous avons d'abord vu que la création même de ces structures dans le cadre du processus des SMSI a fait l'objet d'un manque de consensus sur leur pertinence. Ensuite, la fausse dimension «mondiale » suggérée par les dénominations respectives de ces institutions ainsi que les discours et rhétoriques qu'elles employaient ont créé des attentes démesurées et illusoires auprès des potentiels bénéficiaires de la solidarité numérique en Afrique par rapport aux capacités d'action réelles du FSN et de l'ASN, qui ne disposaient pas des moyens ni humains ni financiers adéquats pour mener à bien leurs programmes. Comme l'a fait remarquer un fonctionnaire du PNUD à Dakar lors de nos entrevues, «faire un télécentre, c'est facile. La difficulté, c'est d'en faire 1000 et 10000 . Et là, il faut non seulement une infrastructure organisationnelle lourde, mais aussi les moyens financiers qui vont $\operatorname{avec}^{22}$ ». Étant donné que la solidarité numérique ne peut être que l'émanation d'un construit social, l'hypothèse d'un décalage entre, d'une part, la perception de l'offre de solidarité numérique telle que proposée par les décideurs politiques et les promoteurs de la société de l'information au Nord et, d'autre part, les caractéristiques de la demande locale des populations du Sud, cibles des politiques internationales de solidarité numérique, est bien vérifiée.

Ce problème de décalage entre l'offre internationale de solidarité numérique et les attentes liées au développement numérique des pays africains est le reflet du manque de stratégies et de vision structurante, des problèmes de mauvaise gestion financière et des dysfonctionnements institutionnels ayant conduit à la dissolution du FSN et de l'ASN.

Il peut aussi être reproché à l'ASN et au FSN d'avoir privilégié des stratégies et des méthodes d'intervention globales, sans cible particulière et dans une approche que l'on 
pourrait qualifier de "one-size-fits-all ${ }^{23}$ ». L'offre de programme d'actions internationales en matière de solidarité numérique paraissait mal structurée, pas assez qualifiée, peu visible et s'inscrivait dans une logique de solutions « clé en main » qui s'avère inadaptée aux spécificités des contextes d'acculturation socioculturels liées à la réception de cette offre localement. Les conditions de mise en œuvre des programmes et des politiques de solidarité numérique, notamment dans le domaine de l'éducation numérique et de la télémédecine, ont révélé l'existence de tensions, de contradictions et de déséquilibre dans le rapport entre donateurs du Nord et assistés du Sud. Les propos que nous avons recueillis lors de notre entrevue avec Ababacar Diop, ancien conseiller spécial du président Wade, chargé de la solidarité numérique et directeur du projet Sénéclic, illustrent bien ce constat :

«Il était prévu que $66 \%$ des projets financés par le FSN soient réalisés en Afrique. Nous, les Africains, étions censés être les grands bénéficiaires de ce programme et, pourtant, nous étions écartés des instances de décision et de gouvernance de ce fonds. Comment peut-on prétendre réaliser des projets pour l'Afrique en restant au Nord, éloigné des réalités du terrain et parachuter, par exemple, des unités de télémédecine ? »"

La solidarité numérique telle qu'elle a été incarnée dans ces programmes internationaux n'a pas véritablement changé l'approche directive et ethnocentriste de la coopération internationale dans le domaine du numérique, qui fait que celui qui donne tend à imposer sa volonté à celui qui reçoit. Ces programmes impliquant des transferts technologiques ont été confrontés aux difficultés inhérentes au poids des pratiques et des héritages institutionnels et culturels nationaux, à l'inadéquation du contexte de réception par rapport aux technologies numériques importées (des technologies parfois assimilées à " un luxe improductif au regard des priorités classiques de développement $\left.{ }^{25} »\right)$, à la résistance locale au changement, au manque de volonté des dirigeants locaux et à l'insuffisance des ressources et des compétences en vue d'une autonomisation des bénéficiaires. Ces derniers sont longtemps restés des récepteurs passifs des TIC, rêvant d'une greffe de modèle de développement basé sur le miracle du rattrapage technologique. Mais la greffe n'a pas pris, et le rattrapage technologique tant promis n'a pas été au rendez-vous en raison de l'incapacité des promoteurs et des acteurs de la solidarité numérique à tenir compte des besoins et des contraintes spécifiques liés à leur terrain d'intervention. Une action de solidarité numérique ne consiste pas simplement à offrir des ordinateurs ou des tableaux numériques et d'attendre que les bénéficiaires s'adaptent à ces équipements. Ce n'est pas aux utilisateurs de s'adapter à la technologie, mais aux donateurs des équipements de s'assurer que l'efficacité de leur action de solidarité numérique dépendra, pour une large part, de la capacité de la technologie à répondre aux besoins et aux attentes.

En revanche, la solidarité numérique décentralisée, menée selon une approche plus territorialisée, s'appuie quant à elle sur la prise en compte des besoins des partenaires et l'implication des populations cibles dans la mise en œuvre du projet. Cette démarche inclusive semble avoir permis à nombre de projets menés dans le cadre de la coopération décentralisée numérique de sortir des pièges de l'assistanat technologique, en rendant un peu plus autonomes les bénéficiaires finaux. Ces succès sont toutefois à relativiser eu égard au fait que la plupart de ces projets restent sectoriels et ponctuels en raison de la durée courte de la période de financement (entre un et trois ans). Certains experts parlent même de "saupoudrage » en raison du caractère hétéroclite, disparate et dispersé des effets de ces projets. Philippe Vidal résume bien la situation : 
«Le concept de solidarité numérique en tant que support des volontés locales manque d'une stratégie partagée avec les partenaires du Sud et reste encore malade de son administration. [...] Faire naître l'innovation dans des environnements sociétaux où les besoins les plus élémentaires (nourriture des populations) ne sont pas toujours garantis reste un défi compliqué et de long terme ${ }^{26} »$.

Le meilleur scénario pour l'émergence d'une véritable solidarité numérique passe non pas par des programmes montés de toute pièce au Nord par des bureaucrates qui n'ont jamais mis un pied en Afrique, mais dépendra définitivement de la généralisation de mouvements, tels que le do it yourself (faites-le vous-même), qui permettraient de voir des jeunes ingénieurs informatiques, développeurs ou hackers ainsi que des start-up, des FabLab, des espaces de coworking donner un véritable coup d'accélérateur à la production et à la diffusion d'innovations numériques et sociales portant le label Made in Africa et implantées sur le continent. La bonne nouvelle est que ce scénario n'est pas de la pure fiction ou un vœu pieu ; il prend déjà forme sous nos yeux avec l'émergence, au cours de ces dernières années, d'un écosystème solidaire et très dynamique d'acteurs africains du numérique qui, de Nairobi à Dakar, en passant par Lagos, Cotonou, Lomé ou Abidjan, développent des applications et des systèmes automatisés de messagerie mobile dans des domaines aussi variés que l'agriculture, l'éducation, la santé, les finances ou encore le tourisme. Par ailleurs, l'Afrique a déjà ses fabricants de tablettes, de Smartphones et même d'imprimantes 3D, et peut dorénavant se permettre de rêver à une solidarité numérique Sud-Sud, de proximité plus horizontale et certainement plus vertueuse et efficace que la solidarité numérique « importée d'ailleurs », qui a longtemps maintenu le continent dans un assistanat trop intéressé du Nord et pas toujours assumé au Sud.

\section{BIBLIOGRAPHIE}

Banque mondiale, World Development Report 2016 : Digital Dividendes, Banque mondiale, Washington, 2016, disponible sur http://www.worldbank.org.

Benamrane Djilali, « Le Fonds de solidarité numérique : un abcès de la gouvernance mondiale en gestation ", dans Benamrane Djilali, Jaffré Bruno, Urvoy Sangharé Marie-Laure, Verschave François-Xavier (dir.), Les Télécommunications, entre bien public et marchandise, Charles-Léopold Mayer, coll. « Dossier pour un débat » (no 148), Paris, 2005, p. 251-266.

Depover Christian, Karsenti Thierry, Komis Vassilis, Enseigner avec les technologies. Favoriser les apprentissages, développer des compétences, Presses de l’Université du Québec, Québec, 2007.

Dieth Alexis, «Les NTIC en Afrique et la problématique du changement des paradigmes par l'éducation numérique ", Aprélia, 2012, disponible sur http://aprelia.org/index.php/fr.

Direction générale de la coopération internationale et du développement, Guide de la coopération décentralisée pour la solidarité numérique, ministère des Affaires étrangères et européennes / Direction générale de la coopération internationale et du développement, coll. « Partenariats », Paris, 2008.

Emerson Robert, « Le travail de terrain comme activité d'observation. Perspectives ethnométhodologistes et interactionnistes », dans Cefaï Daniel (dir.), L’Enquête de terrain, La 
Découverte / Mouvement anti-utilitariste dans les sciences sociales, coll. « Recherches. Bibliothèque du Mouvement anti-utilitariste dans les sciences sociales », 2003, p. 398-424.

Eveno Emmanuel, « Les collectivités locales et les TIC en France : De l'apprentissage de la décentralisation à la découverte de la coopération décentralisée », dans Collectif eAtlas francophone de l'Afrique de l'Ouest, «Sociétés africaines de l'information. vol. 2 : Recherches et actions en Afrique de l'Ouest francophone ", ministère des Affaires étrangères, Paris, 2012, p. 103-118, disponible sur http://www.diplomatie.gouv.fr.

Loukou Alain François, « "Les TIC au service du développement en Afrique.” Simple slogan, illusion ou réalité ? », Tic \& Société, vol. 5, nº 2-3, 2012, disponible sur http:// journals.openedition.org/ticetsociete.

Loum Ndiaga, "Questionner la solidarité numérique : une tentative de construction théorique », dans Agbobli Christian (dir.), Quelle communication pour quel changement ? Les dessous du changement social, Presses de l'université du Québec, coll. « Communication », Québec, 2009, p. 127-142.

Olivier De Sardan Jean-Pierre, La Rigueur du qualitatif. Les contraintes empiriques de l'interprétation socio-anthropologique, Academia-Bruylant, coll. « Anthropologie prospective » ( $\left.n^{\circ} 3\right)$, Louvain-laNeuve, 2008.

Ryfman Philippe, « Extension des solidarités et concentration organisationnelle : le cas des ONG », dans Devin Guillaume (dir.), Les Solidarités transnationales, actes du IIII ${ }^{\mathrm{e}}$ colloque de la section d'études internationales de l'Association française de science politique (Paris, 21-22 octobre 2003), L'Harmattan, coll. « Logiques politiques », Paris/Budapest/Turin, 2004, p. 29-42.

Tchéhouali Destiny, «Les politiques et actions internationales de solidarité numérique à l'épreuve de la diffusion des TIC en Afrique. Bilan et perspectives ", thèse de doctorat, université Toulouse II - Le Mirail, Toulouse, 2013.

Union internationale des télécommunications, « Rapport. Mesurer la société de l'information 2015. Résumé analytique », Union internationale des télécommunications, Genève, 2015a, disponible sur http://www.itu.int/fr.

Union internationale des télécommunications, « ICT Development Index 2015 », 2015b, disponible sur http://www.itu.int/fr.

Vidal Philippe, «L'aide au développement dans le domaine des TIC : entre logiques technomarchandes et charity-business ", dans Nonjon Alain (dir.), L'Afrique des nouvelles convoitises, Ellipses, Paris, 2011, p. 95-96.

\section{NOTES}

1. Banque mondiale, 2016.

2. Union internationale des télécommunications, 2015a.

3. Union internationale des télécommunications, 2015b.

4. Sardan, 2008, p. 11.

5. Au moment de la réalisation de cette recherche, nous avions le statut de doctorant menant une thèse dans le cadre d'une convention industrielle de formation par la recherche (CIFRE) et occupions en même temps le poste de chargé de mission TIC au sein de l'Agence mondiale de solidarité numérique.

6. Emerson, 2003.

7. Emerson, 2003.

8. Loum, 2009, p. 130 
9. Benamrane, 2005 , p. 251

10. Tchéhouali, 2013, p. 338.

11. Ryfman, 2004, p. 41.

12. Du nom de l'une des plus anciennes universités du monde, celle de Tombouctou, au Mali.

13. Dans chaque pays africain où le programme Sankoré est mis en place, une convention (accord de coopération bilatéral) est passée entre la DIÉNA et le Gouvernement (généra- lement représenté par le ministère en charge de l'enseignement primaire ou de la formation professionnelle). À ce jour, seuls six pays ont signé un protocole d'accord avec la DIÉNA : Mali (31 mai 2010), Sénégal (15 octobre 2010), île Maurice (22 décembre 2010), Madagascar (juillet 2011), Burkina Faso (septembre 2011) et Bénin (24 mai 2012).

14. Décret $n^{\circ} 2010-85$ du 22 janvier 2010 instituant un délégué interministériel pour l'éducation numérique en Afrique,

http://www.legifrance.gouv.fr/affichTexte.do?

cidTexte=JORFTEXT000021725739\&categorieLien $=$ id

15. http://innovafrica.org/fr/project/programme-sankore

16. Dieth, 2012.

17. Interview réalisée en février 2009 sur le réseau Web2solidarité.org, dans le cadre d'un débat participatif sur l'éducation numérique en Afrique animé par Destiny Tchéhouali.

18. Entrevue de terrain, réalisée par Destiny Tchéhouali en 2011.

19. Depover, Karsenti, Komis, 2007.

20. Source : Document d'archives de l'Agence mondiale de solidarité numérique.

21. Eveno, 2012, p. 119.

22. Entrevue de terrain en 2011.

23. Se traduit littéralement par « taille unique ", contraire à une approche de projet sur mesure.

24. Entrevue de terrain en 2011.

25. Loukou, 2012, p. 49.

26. Vidal, 2011, p. 95-96.

\section{RÉSUMÉS}

Ce texte dresse un bilan des politiques internationales dans le domaine du développement numérique des pays du Sud en examinant les initiatives de solidarité numérique menées entre 2009 et 2013 par l'Agence (ASN) et le Fonds mondial de solidarité numérique (FSN) en Afrique. L'analyse s'appuie sur une démarche d'observation participante réalisée dans un contexte de recherche-action ainsi que sur des études de cas spécifiques de projets d'éducation numérique et de télémédecine, permettant de faire ressortir les écarts significatifs et les contradictions entre d'une part les discours des institutions promotrices de la solidarité numérique internationale et les objectifs de leurs projets ou programmes et d'autre part les attentes des publics bénéficiaires et les résultats concrets obtenus à l'issue de l'implémentation de ces projets sur le terrain.

This paper addresses the issue of international policies in the field of digital development in developing countries by examining digital solidarity initiatives conducted between 2009 and 2013 by the World digital solidarity Agency (DSA) and the World digital solidarity Fund (DSF) in Africa. Our analysis is based on a participatory observation process carried out in a research-action context as well as on specific case studies of projects in digital education and telemedicine which 
highlight the significant differences and contradictions between on the one hand, the discourses of the institutions promoting international digital solidarity and the objectives of their projects, and on the other hand the expectations of the beneficiary publics and the concrete results obtained after the implementation of these projects in Africa.

INDEX

Mots-clés : politiques internationales, développement numérique, Afrique, solidarité

Keywords : international policies, digital development, Africa, solidarity

\section{AUTEUR}

DESTINITY TCHÉHOUALI

ISOC Québec 